$\Delta$ Palabras clave/ Asentamientos humanos, participación comunitaria, hábitat, diseño urbano.

$\Delta$ Keywords/ Human settlements, community participation, habitat, urban design.

$\Delta$ Recepción/ 18 abril 2017

$\Delta$ Aceptación/ 12 julio 2017

\title{
Ciudades de barro. Asentamiento informal Fundadores Bajo de Armenia, Colombia ${ }^{1}$
}

\author{
Mud cities. Fundadores Bajo informal \\ settlement in Armenia, Colombia ${ }^{1}$
}

RESUMEN/ Se presenta una investigación participativa entre academia y comunidad con el fin de encontrar posibles acciones de mejoramiento urbano-arquitectónico para el asentamiento informal Fundadores Bajo en la ciudad de Armenia, Colombia, que propicien la transformación de un territorio en el que habitan más de cincuenta familias. La investigación se enfoca en el estudio, diagnóstico, formulación y propuesta, considerando características sociales, económicas y de infraestructura presente. Para ello, se llevaron a cabo entrevistas, encuestas, fichas de recolección de datos y ejercicios de cartografía social en los que los habitantes del asentamiento expusieron las deficiencias en su hábitat y, a su vez, sueños e ideales colectivos para el mejoramiento del mismo. El estudio permitió conocer las condiciones de vida de los habitantes, quienes son víctimas de la exclusión social y de falta de planeación gubernamental. Asimismo, se produjo un diagnóstico situacional comunitario y dos propuestas urbano-arquitectónicas planteadas de forma participativa para el corto y largo plazo. ABSTRACT/ This work presents a participatory research involving the academia and the community aimed at finding potential urban-architectural improvement measures for the Fundadores Bajo informal settlement in the city of Armenia, Colombia. The goal is to promote the transformation of an area home to more than 50 families. The research consists on a study, diagnostic, formulation and proposal, also addressing existing social, economic and infrastructure characteristics. For this purpose, interviews, surveys, data sheet collection and social mapping exercises were conducted whereby the settlement's inhabitants explained the habitat's weaknesses and, at the same time, their common dreams and expectations regarding its improvement. The study provided insights on the living conditions of a population subject to social exclusion and lack of government planning. Likewise, a community situational diagnostic was made and two long and short-term urban-architectural proposals were submitted in a participatory fashion.

INTRODUCCIÓN. Se presentan resultados investigativos de las condiciones físicas y de habitabilidad presentes en el territorio del asentamiento humano informal Fundadores Bajo en Armenia, Colombia, que sirvieron como base para la formulación de dos propuestas urbano-arquitectónicas dirigidas al mejoramiento barrial en dicho lugar. Todo ello a partir del diagnóstico y diseño participativos con la comunidad, iniciativa surgida desde la fundamentación metodológica y conceptual de la Facultad de Arquitectura de la Universidad La Gran
Colombia Seccional Armenia, Colombia, con el aporte de las identidades, necesidades y sueños de los habitantes del lugar, liderados por la Junta de Acción Comunal (JAC) del asentamiento.

Fundadores Bajo está ubicado en un sector que cuenta, en su contexto urbano, con equipamientos de educación, comercio y salud, situación que históricamente ha favorecido su consolidación urbanística. El mismo está compuesto por cerca de cincuenta familias y hace parte de la creciente problemática de habitabilidad informal que se identifica en el Municipio de Armenia, donde existen más de 40 asentamientos informales y alrededor de 14.000 personas que se localizan principalmente en zonas de riesgo de desastres por remoción de masa o de protección ambiental (Alcaldía de Armenia 2008). Fundadores Bajo posee infraestructura física deficiente, no cuenta con zonas para la divulgación sociocultural de la población, carece de regulación necesaria para una adecuada relación con el entorno y se encuentra vulnerable ante la posibilidad de deslizamientos de tierra.

'Este artículo es producto del proyecto de investigación: "Alternativas de mejoramiento urbano incluyente del asentamiento humano informal Fundadores Bajo de la ciudad de Armenia"; financiado por a Universidad La Gran Colombia Seccional Armenia y desarrollado en el 2016 con el apoyo de María del Mar Cancino y Yenny Yulieth Suarez, de la Facultad de Arquitectura de la mencionada universidad. Asi mismo, hace parte de los resultados que se arrojan en uno de los sectores de estudio del proyecto de investigación doctoral "Interpretaciones desde abajo. Una lectura a la lógica de la ciudad informal presente en intersticios urbanos conquistados", que el autor adelanta en el Doctorado en Estudios Territoriales de la Universidad de Caldas - Colombia. 
La presente investigación tuvo como objetivo proponer acciones enfocadas al mejoramiento integral del asentamiento humano informal Fundadores Bajo por medio de la descripción, análisis y formulación participativa de estrategias dirigidas al desarrollo urbano y la transformación del territorio. En su desarrollo metodológico se utilizó el método Territorii (Bozzano 2009) para el abordaje de realidades territoriales, y el método Change by Design (Apsan, Anthony y Nuñez 2011) en la formulación participativa y sostenible de propuestas. De esa manera, se aplicaron técnicas mixtas, ya que se utilizaron indicadores numéricos e interpretativos en tres fases: (1) Identidades, donde se realizó una aproximación y documentación del sector, con la que se logró conocer su contexto histórico y su situación actual aplicando entrevistas y recorridos de campo con los habitantes del lugar, permitiéndole al equipo investigador reconocerlo y ser reconocidos por la comunidad (imagen 1); (2) Necesidades/ Oportunidades, durante la cual se realizó un diagnóstico situacional del asentamiento informal por medio del análisis de información primaria y secundaria, a partir de fichas de recolección de información, encuestas y talleres participativos donde se analizaron aspectos normativos, sociales y de infraestructura física; y (3) Sueños de la comunidad y su entorno, generando una simbiosis entre las distintas variables y resultados previos, y donde se formularon de manera colectiva ideas y criterios de diseño urbano-arquitectónico que concibieran la apropiación y mejoramiento del asentamiento.





Imagen 1. Socialización inicial del proceso investigativo con la comunidad (fuente: El autor).

El desarrollo de las fases mencionadas contó con la participación activa de la comunidad, siendo esta fuente primaria de información y con quien se realizaron jornadas de ejercicios participativos y devolución permanente de resultados, en consonancia con los postulados metodológicos de la Investigación-Acción-Participación (IAP), en donde se genera conocimiento que promueve los cambios que las comunidades requieren (Fals Borda 1993). Con el estudio se establecieron las condiciones de vida de los habitantes; se produjo un diagnóstico situacional comunitario y dos propuestas urbano-arquitectónicas planteadas de forma participativa para el corto y largo plazo.

\section{IDENTIDADES. Localización.}

El asentamiento humano informal Fundadores Bajo está ubicado en la zona centro-norte de la ciudad de Armenia, entre la carrera 18 con calle 2 (imagen 2). Pertenece a la comuna 10, cuenta con accesibilidad desde las calles 1 y 2 (imagen 3) y se localiza cerca de vías principales de la ciudad, situación que favorece la interacción de sus habitantes con el contexto y ha propiciado históricamente la consolidación

\section{del mismo.}

Historia. Los primeros habitantes de Fundadores Bajo llegaron en los años 50 y provenían del departamento del Tolima. El sector era conocido, en esa época, como las 60 casas sobre la calle 2 con carrera 18, por donde se trazó la vía férrea para conectar la ciudad con el centro del país, la misma que nunca pudo entrar en funcionamiento generando espacios de ciudad que posteriormente fueron apropiados por nuevos habitantes. En 1963 se construyó, cerca del lugar, el Parque Fundadores, un espacio público que se convirtió en punto de referencia para la ciudad y, a partir de ese momento, el asentamiento se consolidó y reconoció como Fundadores Bajo. Hacia el año 1970, con ayuda de la alcaldía de Armenia y la JAC, algunas familias fueron reubicadas en otras en zonas de la ciudad.

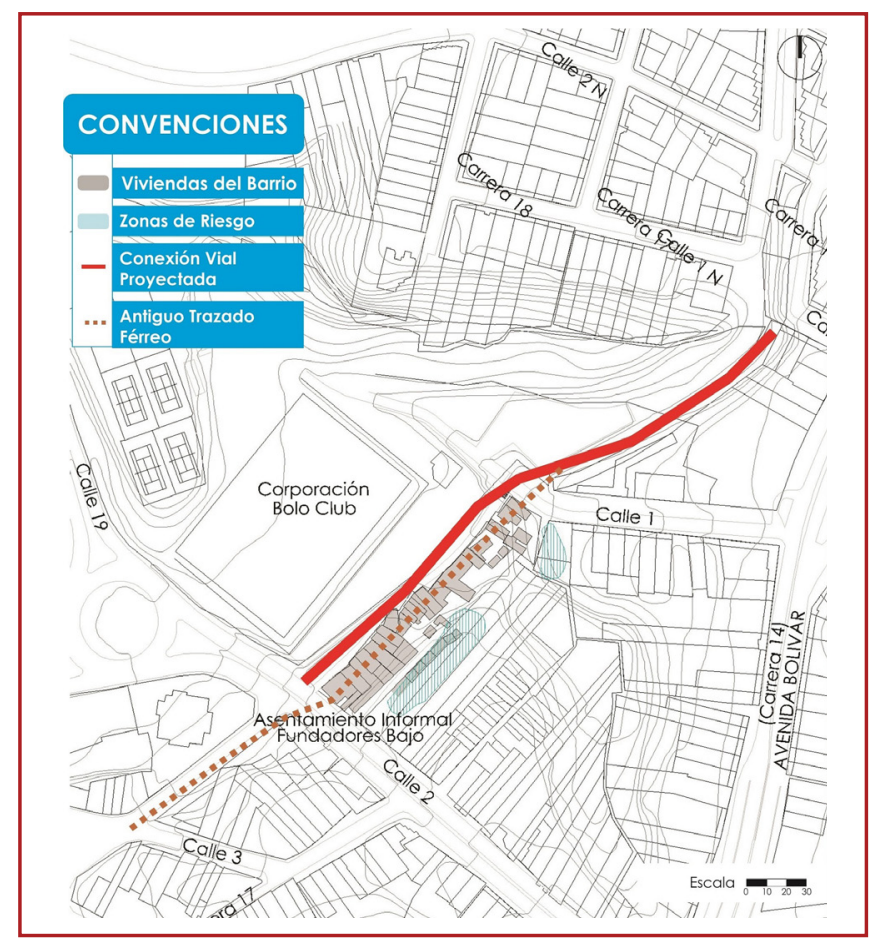

Imagen 3. Condiciones urbanisticas presentes en el asentamiento (fuente: Elaboración propia). 
Quienes se quedaron continuaron con el crecimiento inadecuado del asentamiento. Actualmente existen en el lugar 35 edificaciones (imagen 3), y en ellas habitan 52 familias sobre un área aproximada de $5.410 \mathrm{~m}^{2}$, un reflejo de la búsqueda de sus

\section{habitantes a ejercer su derecho a la ciudad.}

NECESIDADES Y OPORTUNIDADES. Diagnóstico normativo.

A partir de la revisión normativa realizada, se identificó que el suelo donde se localiza el asentamiento hace parte de la antigua línea férrea, propiedad del Fondo Pasivo Social de Ferrocarriles Nacionales. En la ciudad de Armenia, gran parte de este tipo de predios que entraron en desuso han dado lugar al surgimiento espontáneo de asentamientos humanos, situación que dificulta la titulación de viviendas y la aplicación de una política clara en materia de mejoramiento barrial.

Se encontró también que en el actual Plan de Ordenamiento Territorial (POT) (Concejo Municipal de Armenia 2009) se establece el sector del asentamiento dentro del tipo Residencial cotidiano de redesarrollo y el Redesarrollo como el tratamiento urbanístico al que se debe acoger cualquier iniciativa que allí se plantee. De igual manera, el POT define que al interior de los predios que conforman el asentamiento existe un porcentaje bajo demarcado como Zona de Riesgo de Deslizamiento Cualitativamente Moderado, es decir, se deben ejercer acciones previas de mitigación para realizar cualquier actuación urbanística sobre el mismo.

La mayoría de las familias no cuentan con un título de propiedad del terreno, solo tienen boletos de compraventa y constancia de posesión de mejora, lo que no otorga titularidad sobre el predio ni su edificación. Sin embargo, para las empresas que proveen servicios básicos en la ciudad, esta tenencia informal no ha sido impedimento para la instalación de algunos de los servicios públicos domiciliarios.

\section{Diagnostico multidimensional2.}

A partir de la aplicación de entrevistas y encuestas en las viviendas del asentamiento, se resalta, entre otros, que los niveles de escolaridad de sus habitantes son en un $4 \%$ técnico, $5 \%$ tecnológico y $3 \%$ universitario; el porcentaje restante tiene hasta educación secundaria. Un 75\% de la población encuestada manifestó que habita este lugar por la necesidad de contar con una vivienda propia, sin ser conscientes de que dicha ocupación es ilegal y dicen no contar con los recursos para vivir en un lugar mejor; el $25 \%$ de la comunidad es consciente de que habita el asentamiento de manera ilegal y manifiesta que entre las razones para estar allí se encuentran la cercanía de las familias y la cercanía del lugar con respecto al centro de la ciudad y a sitios de trabajo, estudio, salud y diversión.

Una de las problemáticas principales manifestada por los habitantes es la situación de empleabilidad presente en la población laboralmente activa, donde solo el $22 \%$ de los encuestados dice tener un empleo fijo. Por otra parte, se encontró que en el $47 \%$ de los hogares los ingresos oscilan entre los 201.000 y 400.000 pesos mensuales, evidenciando baja capacidad de ahorro de las familias. Los medios de transporte más utilizados hacia sus lugares de estudio o trabajo son, en orden de preferencia, a pié, bicicleta y bus, lo que denota la cercanía a sus destinos de mayor frecuencia.

Asimismo, se evidenció que tan solo el 31\% de las familias participa de las actividades que se desarrollan con promoción de la JAC, siendo ésta la única organización comunitaria reconocida por los encuestados. Los estudios realizados indican que el $63 \%$ de los habitantes de Fundadores Bajo considera la relación con sus vecinos como buena. Sin embargo, cerca del $42 \%$ no se siente acogido por el resto de la comunidad. Entre los habitantes se encuentran agricultores, jardineros, artesanos, obreros, estilistas, vendedores, entre otros. El 61\% de ellos dijo tener la intención de generar su propio emprendimiento con el fin de aumentar sus ingresos económicos.

\section{Diagnóstico de infraestructura física.}

En este componente del diagnóstico se tomó como referente metodológico el programa de Mejoramiento Integral del Hábitat para la Región Metropolitana del Valle de Aburrá (Colombia), elaborado entre la Universidad Pontificia Bolivariana de Medellín (UPB) y el Área Metropolitana del Valle de Aburrá (2007)3. De esa manera, se pudo determinar, entre otros aspectos, que gran parte de las viviendas de Fundadores Bajo se encuentran construidas con materiales provisionales como guadua, esterilla, madera y zinc. Aunque en su mayoría se identificaron como en riesgo medio en cuanto a vulnerabilidad física, la característica de sus materiales hace contemplar la posibilidad de incendios para un $79 \%$ de las viviendas (imagen 4).

Los resultados también arrojaron que el $66 \%$ de las viviendas se encuentran con posibilidades de colapso y, en ese mismo porcentaje, de sufrir fuertes consecuencias en caso de vendavales. Se concluye que los principales factores de vulnerabilidad frente a un posible riesgo son la materialidad de las construcciones, la falta de acceso vehicular adecuado en casos de emergencia junto con la ausencia de planes preventivos comunitarios y, en casos aislados, la cercanía a zonas de ladera.

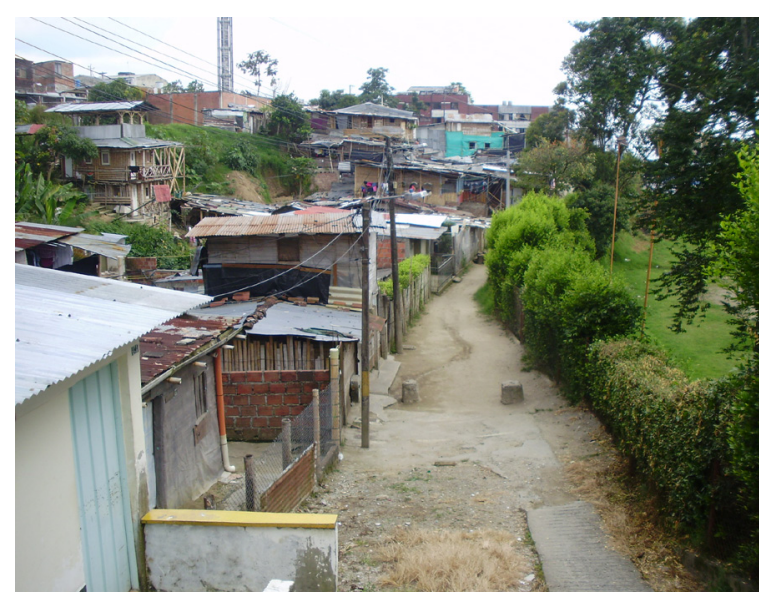

Imagen 4. Panorámica del asentamiento Fundadores Bajo (fuente: El autor). 
El tipo de vivienda ${ }^{4}$ predominante que habitan las familias encuestadas es el de casa en un $90 \%$ y habitación en un $10 \%$, y en promedio las familias están conformadas por 4,5 personas. Con relación al hacinamiento habitacional ${ }^{5}$, éste ocurre en el 19\% de las viviendas. Además, el 39\% de ellas cuenta con espacios compartidos para sanitario, cocina, lavar ropa y dormitorio. Asimismo, en el 39\% de las viviendas no existe una ventana en cada cuarto destinado para dormir, lo que dificulta una adecuada ventilación, propicia escenarios de violencia doméstica y expone a la población a enfermedades, disminuyendo así la calidad de habitabilidad y el desarrollo adecuado de los niños.

El servicio público de recolección de basuras, al igual que el suministro de energía eléctrica, se encuentra en el $100 \%$ de los hogares del asentamiento, de los cuales el $78 \%$ tiene contador de energía individual y el $22 \%$ comparte el suministro. El servicio de acueducto solamente está instalado de manera individual en el $68 \%$ de las viviendas, el $29 \%$ lo recibe de manera compartida y el 3\% no tiene este servicio. La red de alcantarillado cubre el $97 \%$ de las viviendas. En cuanto a espacios libres y de uso común, se identificaron $360 \mathrm{~m}^{2}$ de espacios libres y una caseta comunal construida de manera improvisada. Asimismo, se evidencia la ausencia de alumbrado público en varias zonas del asentamiento, lo que ha generado focos de inseguridad.

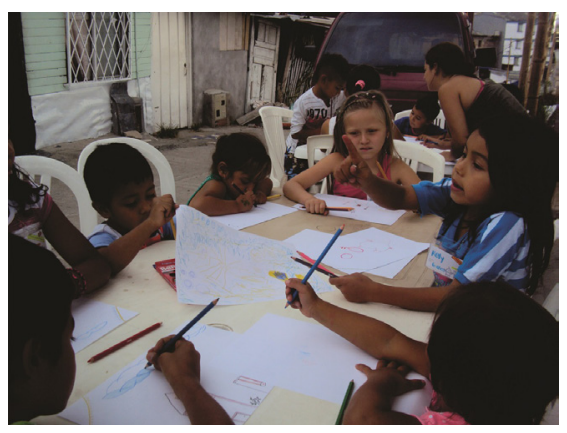

Imagen 5. Taller de dibujo y color (fuente: El autor)
Diagnóstico participativo. Se aplicaron métodos de diseño participativo tomando como referencia la metodología Change by Design (Apsan, Anthony y Nuñez 2011) propia de la Organización No Gubernamental Arquitectos Sin Fronteras del Reino Unido (ASF-UK). A partir de ejercicios dialógicos con grupos focales de la comunidad y actividades como el taller Dibujo y Color: Cómo quiero mi barrio (imagen 5) y el ejercicio de "Deseos para el Barrio en Navidad", realizados con niños, sumado al Taller de Cartografía Social desarrollado con adultos (imagen 6), fue posible establecer, entre otros aspectos, que los lugares favoritos de los niños son la calle principal, la caseta comunal y el polideportivo del barrio vecino. Se evidencia, a partir de lo anterior, la deficiencia de espacios de esparcimiento y recreación para la comunidad, limitándose a los mencionados y a sus viviendas. Con la actividad de Cartografía Social realizada con 12 adultos habitantes del asentamiento, se logró identificar que en los últimos 20 años éste ha mejorado sus condiciones físicas. Como ejemplos de ello mencionaron el acceso principal sobre la calle 2 (que aún sigue siendo restringido para vehículos de mediano tamaño), la calidad constructiva de algunas viviendas y el suministro de servicios públicos. Ellos son conscientes de que estas mejoras se han dado de manera irregular.

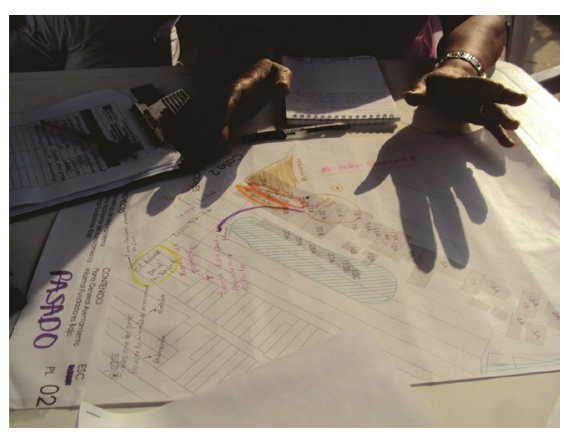

Imagen 6. Taller de cartografía social (fuente: El autor)
De la misma forma, manifestaron que la legalización del asentamiento es una prioridad, que lleva un largo tiempo sin lograrse, dado que aún no se cuenta con los estudios urbanísticos necesarios ni con espacios adecuadas para uso comunitario, y es por esto último que se manifiesta la necesidad de construir una caseta comunal que les permita llevar a cabo diferentes actividades. Otras falencias evidenciadas en dicha actividad son la falta de comunicación entre vecinos que fortalezca la convivencia, la inexistencia de alumbrado público por uno de los accesos, la deficiente materialidad de algunas viviendas que expone a algunas familias a condiciones de riesgo, la falta de canalización de aguas lluvia y la necesidad de reforzamiento de estructuras en viviendas.

Sueños. Finalmente, con este proceso de diseño participativo por medio de actividades de dibujo, juegos, diálogos, entrevistas y el vínculo comunitario, se logró trabajar en propuestas proyectuales a través de planos imaginarios que permitieron identificar ideas urbano-arquitectónicas, en las cuales se incluyeron: mejoras estructurales de las viviendas, diseño de la caseta comunal, implementación de juegos infantiles y mobiliario urbano, prevención del riesgo a deslizamientos, adecuación de recorridos naturales, pavimentación de vía peatonal, integración de los sectores del asentamiento con zonas verdes, estancias de descanso y señalización.

Para ello, luego del análisis de resultados obtenidos, se elaboraron técnicamente las propuestas urbano-arquitectónicas, las cuales están concebidas con la temporalidad y posibilidad de su ejecución por parte de diferentes actores en programas de mejoramiento integral de barrios. Las mismas se concibieron teniendo como referencia la norma urbana establecida para la ciudad y se plantearon tanto para espacios colectivos como para espacios privados en dos escenarios de ejecución: el primero, a corto plazo, con el fin de viabilizar la legalización del asentamiento $y$, el segundo, a largo plazo, una vez que se puedan realizar intervenciones en el lugar. 


\section{PROPUESTAS. Corto plazo.}

Esta propuesta contempla la relocalización de algunas viviendas por hallarse en zonas de riesgo, aumentando así el área destinada para equipamientos colectivos. Esta medida facilita el proceso de legalización, dado que permite la adecuación de $507 \mathrm{~m}^{2}$ de espacios públicos superando el porcentaje mínimo exigido por la normativa para tales efectos. Asimismo, esta propuesta plantea un jardín lineal con arborización característica de la región de forma paralela a la futura vía que se construirá. Esto permitirá cambiar la imagen del lugar (imagen 7).

Complementando las zonas mencionadas, y con el fin de fomentar la apropiación del lugar, se plantea una huerta comunitaria que permita satisfacer algunas necesidades alimentarias de los hogares, dispuesta al lado de la caseta comunal, en la cual será posible el desarrollo de actividades barriales, reuniones educativas, salón de emprendimiento, fiestas y eventos, entre otros. Además, se propone un parque infantil contiguo (imagen 8).

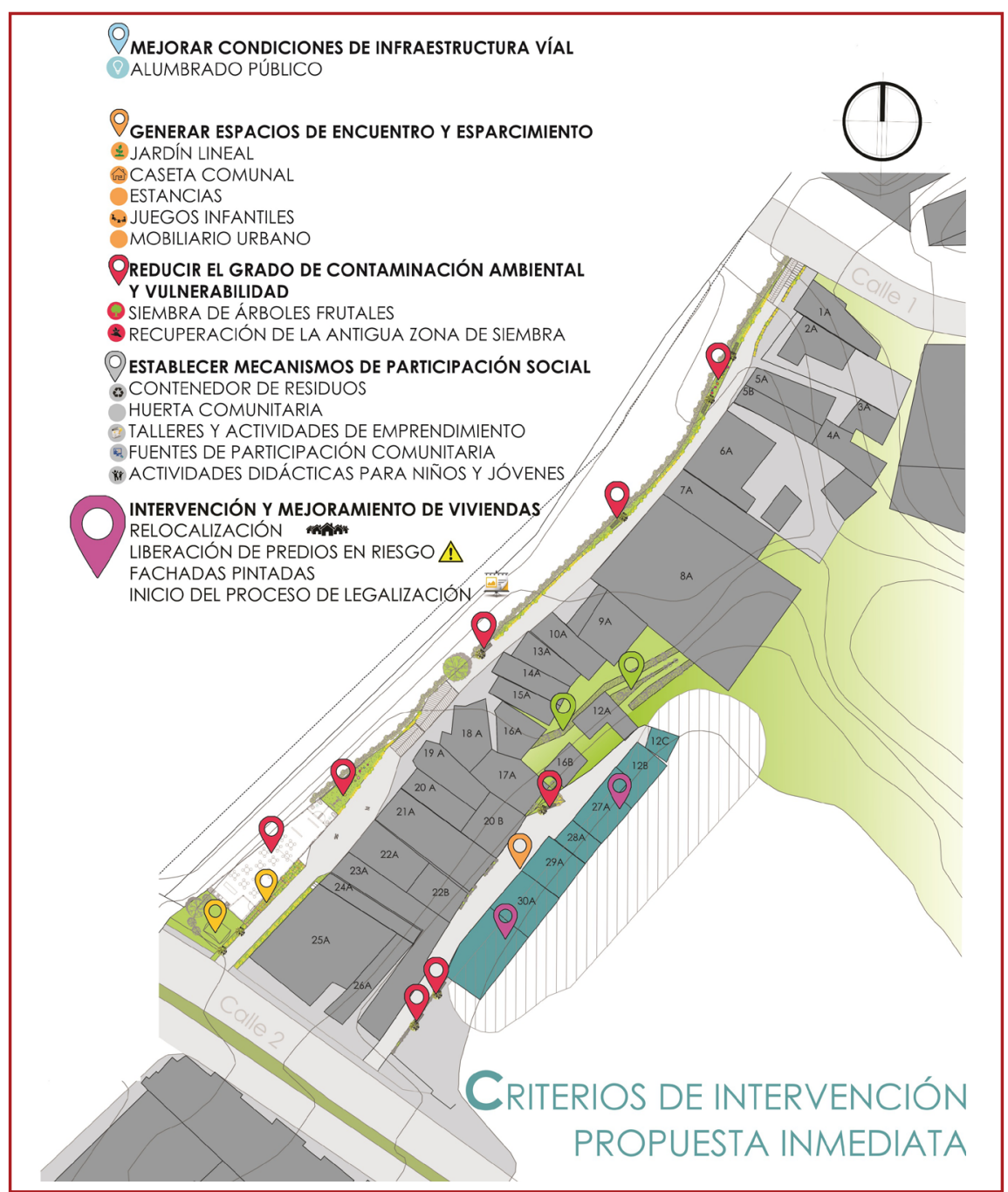

Imagen 7. Planta general criterios de intervención propuesta l (fuente: Elaboración propia).

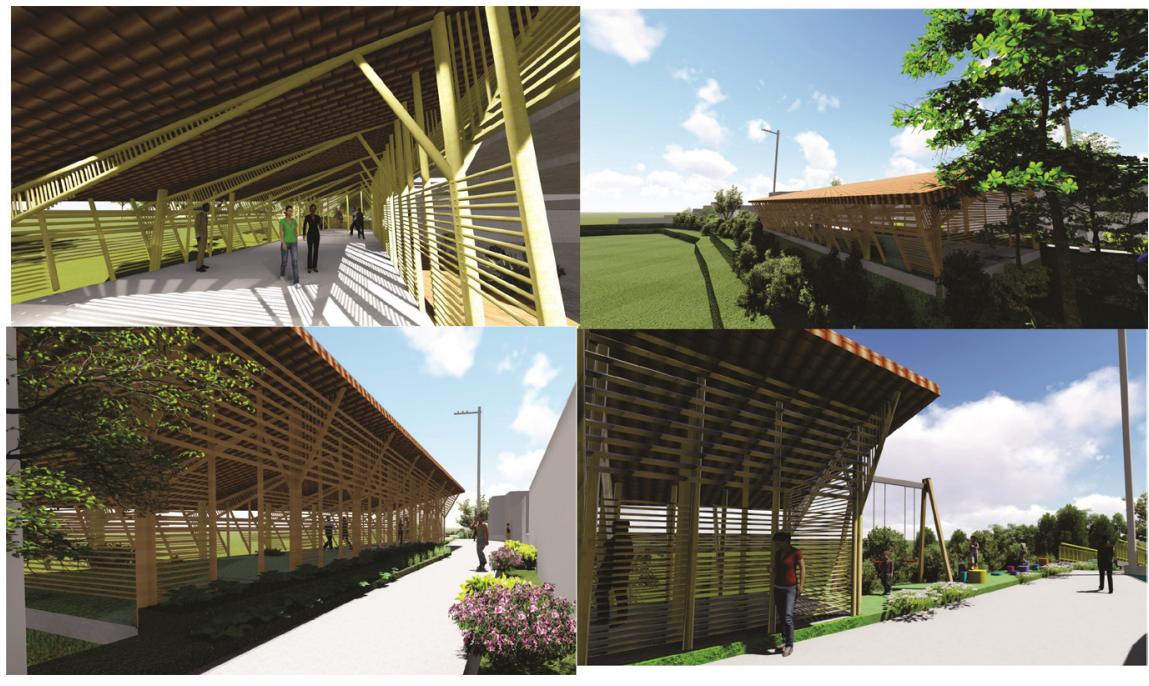

Imagen 8. Perspectivas de la caseta comunal propuesta (fuente: Elaboración propia). 
Largo plazo. La segunda propuesta plantea (de manera complementaria a la anterior) la adecuación del acceso vehicular y parqueaderos para el uso de la comunidad, según la normativa urbana que rige al sector (imagen 9). Además, algunas de las viviendas serán mejoradas en aspectos estructurales y de fachada, mientras que otras serán relocalizadas dentro del mismo asentamiento facilitando su densificación habitacional. Para ello se proponen nuevas edificaciones de cinco niveles de altura con apartamentos de $70 \mathrm{~m}^{2}$ que alberguen de manera digna a todos los actuales habitantes de la comunidad. A nivel urbano, esta propuesta promueve la creación de zonas que fortalezcan la conectividad entre los habitantes y espacios de estancia que complementen recorridos naturales. De manera similar, se sugiere la ubicación del salón comunal en un área central, con capacidad para más de 50 personas, dispuesto para múltiples eventos. A su alrededor se plantea la ubicación de la huerta comunitaria y un parque infantil, los cuales permitirán que las personas se involucren en las dinámicas del lugar y adquieran mayor apropiación. Finalmente, para conservar la memoria del lugar, se propone recuperar antiguas zonas de siembra, cultivadas anteriormente por algunos pobladores.

\section{PROPUESTA PROYECTADA}


Imagen 9. Planta general propuesta proyectada (fuente: Elaboración propia). 
CONCLUSIONES. La producción social del hábitat (Ortiz 2012), entendida como aquellos procesos generadores de espacios habitables, en ocasiones de forma individual y espontánea, que para este caso de estudio se realizó de manera informal sobre un terreno de ciudad en desuso, ha sido el elemento fundamental en la vida de los habitantes de Fundadores Bajo. Todos, de una u otra forma, han sido víctimas de algún tipo de desplazamiento, bien sea por conflicto o por falta de recursos económicos. Por lo tanto, las decisiones de diseño propuestas se han tomado teniendo en cuenta la familiaridad de las personas con su actual entorno de vida en el que han obtenido protección y bienestar.

A lo largo de su historia, la localización de Fundadores Bajo ha facilitado la inclusión de sus habitantes en las dinámicas urbanas. Éstos consideran que su hábitat no se limita solo a su lugar de residencia sino a los espacios y equipamientos que la ciudad ofrece y a los que pueden acceder -en un hecho de equidad frente a la 'ciudad formal' que los circunda-, razón por la cual los planes de reubicación del asentamiento que han promovido algunas entidades no son considerados por ellos como una acertada solución a su situación de ilegalidad. Por otra parte, las alternativas y estrategias de mejoramiento urbano y barrial propuestas a lo largo del proyecto, responden a las necesidades expresadas por la comunidad a través de la comunicación, el diálogo e inclusión, para así posibilitar impactos positivos en el sector. Sin embargo, la falta de responsabilidad social en los profesionales involucrados en las condiciones de hábitat humano para capacitar y acompañar a las comunidades en los procesos internos, sumada a la ausencia de operatividad de los entes territoriales para afrontar la problemática de ilegalidad del asentamiento, ocasionan baja efectividad en los instrumentos de gestión contemplados en la Ley 388 de 1997 (Congreso de la República de Colombia 1997), que permitirían la ejecución y sostenibilidad de las mismas.
Finalmente, para fortalecer este tipo de emprendimientos sociales, se considera necesario contar con el apoyo, inclusión y participación de la academia desde diversas disciplinas, contribuyendo no solo a la comunidad del asentamiento sino a otros sectores de la ciudad en condiciones similares, aunando esfuerzos que fomenten el mejoramiento de la calidad de vida y la sana convivencia. De la misma forma, es importante promover la comunicación entre los habitantes y el gobierno local, de manera tal que estudios sobre las dinámicas territoriales se hagan con mayor profundidad, dada la falta de promoción de los programas de apoyo desde los organismos del Estado, los que podrían ser destinados al mejoramiento de barrios de origen informal con posibilidades de legalización, como es el caso de Fundadores Bajo. $\mathbf{\Delta u}$

\section{REFERENCIAS}

Alcaldía de Armenia, 2008. "Diagnóstico municipal". En Alcaldía de Armenia, Plan de Ordenamiento Territorial de Armenia 2009-2023. Armenia: Alcaldia de Armenia.

Apsan, A, Anthony, M. y Nuñez, I., 2011. Change by design. Building communities through participatory design. Nueva Zelanda: Urban Culture Press.

Bozzano, H., 2009. "El Método Territorii. Una mirada territorial a proyectos e investigaciones no siempre territoriales." 8th International Conference of Territorial Intelligence. ENTI. 4-7 de noviembre de 2009, Salerno. Italia.

Concejo Municipal de Armenia, 2009. Acuerdo Municipal No 019 de Noviembre de 2009 por medio del cual se aprueba el Plan de Ordenamiento Territorial del Municipio de Armenia, para el periodo 2009-2023. Armenia: Gaceta Municipal de Armenia.
Congreso de la República de Colombia, 1997. Ley 388. Bogotá D.C.: Diario Oficial No 43127, de 24 de julio de 1997.

Fals Borda, O., 1993. "La investigación participativa y la intervención social." Documentación social, 92, 9-22. Ortiz, E., 2012. Producción social de la vivienda y el hábitat. Bases conceptuales y correlación con los procesos habitacionales. Mexico D.F.: Habitat International Coalition.

Universidad Pontificia Bolivariana de Medellin y Área Metropolitana del Valle de Aburrá, 2007. Mejoramiento Integral del Hábitat para la Región Metropolitana del Valle de Aburrá. Consolidación de una metodología. Medellin: UPB. 